\title{
HDAC6-selective inhibitors enhance anticancer effects of paclitaxel in ovarian cancer cells
}

\author{
JUNG YOO ${ }^{1}$, YU HYUN JEON ${ }^{1}$, DONG HOON LEE ${ }^{1}$, GO WOON KIM ${ }^{1}$, SANG WU LEE ${ }^{1}$, \\ SO YEON KIM ${ }^{1}$, JONGSUN PARK ${ }^{2,3}$ and SO HEE KWON ${ }^{1,4}$
}

\author{
${ }^{1}$ Department of Pharmacy, College of Pharmacy, Yonsei Institute of Pharmaceutical Sciences, Yonsei University, \\ Incheon 21983; ${ }^{2}$ Department of Pharmacology, College of Medicine; ${ }^{3}$ Department of Medical Science, \\ Metabolic Syndrome and Cell Signaling Laboratory, Institute for Cancer Research, College of Medicine, \\ Chungnam National University, Daejeon 35015; ${ }^{4}$ Department of Integrated OMICS for Biomedical Science, \\ Yonsei University, Seoul 03722, Republic of Korea
}

Received October 13, 2020; Accepted January 4, 2021

DOI: 10.3892/ol.2021.12462

\begin{abstract}
Histone deacetylase 6 (HDAC6)-selective inhibitors are potent anticancer agents that are gaining increasing attention and undergoing various developments. These have been approved or are under clinical trials for use with other anticancer agents, such as pomalidomide, anti-programmed death-ligand 1 antibody and paclitaxel, for various types of cancer, including solid tumors. In the present study, a second generation HDAC6-selective inhibitor, ACY-241 (citarinostat), and a novel inhibitor, A452, exhibited synergistic anticancer effects with paclitaxel in AT-rich interaction domain 1A-mutated ovarian cancer in vitro. Co-treatment of paclitaxel and the two HDAC6 inhibitors synergistically decreased cell growth and viability of TOV-21G. Furthermore, the protein expression levels of pro-apoptotic markers, such as poly(ADP-ribose) polymerase, cleaved caspase-3, Bak and Bax, were increased, whereas the expression levels of anti-apoptotic markers, such as Bcl-xL and Bcl-2, were decreased synergistically. Treatment with all drug combinations increased the portion of apoptotic cells in fluorescence-activated cell sorting analysis. These results demonstrated synergy between paclitaxel and HDAC6-selective inhibitors, providing further impetus for clinical trials of combination therapy using HDAC6-selective inhibitors, not only in ovarian cancer but also in other tumors.
\end{abstract}

\section{Introduction}

Ovarian cancer is the second leading cause of female reproductive cancer death. In 2018, ovarian cancer affected

Correspondence to: Professor So Hee Kwon, Department of Pharmacy, College of Pharmacy, Yonsei Institute of Pharmaceutical Sciences, Yonsei University, 85 Songdogwahak-ro, Yeonsu-gu, Incheon 21983, Republic of Korea

E-mail: soheekwon@yonsei.ac.kr

Key words: histone deacetylase 6, ACY-241, citarinostat, A452, ovarian cancer, paclitaxel, combination therapy approximately 295,414 women worldwide, with a predilection for postmenopausal women (1). Ovarian cancer carries a poor prognosis, with a 5-year survival rate of only $30 \%$ and deaths occurring within just 2 years of diagnosis. This low survival rate can be attributed to the late diagnosis of the disease. A significant proportion of women are diagnosed with ovarian cancer at advanced stages, and despite initial complete responses to chemotherapy, $70 \%$ of patients develop recurrence within 18 months (2). Current treatment methods for ovarian cancer are surgery and cytotoxic chemotherapy. When complete surgical debulking seems difficult for the patient, pre- or postoperative chemotherapy may be used as an additional therapeutic strategy. For the past 40 years, cytotoxic chemotherapy based on platinum- and taxane- group drugs such as carboplatin, cisplatin, and paclitaxel (PTX), have been used for first-line chemotherapy. However, despite several clinical trials, regimens containing platinum have not shown improvements for the past 20 years largely due to drug toxicity and resistance. In fact, of the $80 \%$ of patients with good initial responses to standard treatments, $70 \%$ experience disease recurrence (1). In addition to the problem of drug-resistance, drug-refractory patients exhibit the worst prognosis for treatment (2). Patients whose disease progress despite drug treatment have been managed with pegylated doxorubicin or topotecan, both of which have low response rates. Therefore, a novel strategy identified through molecular characterization is required to overcome drug-resistance and to offer new treatment options for patients with advanced and relapsed ovarian cancer.

PTX is a taxane-based, microtubule-stabilizing drug that has been approved by the Food and Drug Administration (FDA) for the treatment of various types of solid cancers, including those of the breast, lung, and ovary (3). PTX inhibits normal dissociation of $\alpha$ - and $\beta$-tubulin subunits, thereby increasing the percentage of tubulin subunits that polymerize into microtubules (4). As a result, PTX reduces the concentration of free-tubulin subunits necessary for microtubule assembly, causing the mitotic arrest of cancer cells. Despite the efficacy of PTX in killing cancer cells, resistance remains a major obstacle to overcome. There are numerous molecular mechanisms by which cancers evade the cytotoxic effect of PTX. 
These mechanisms include, but are not limited to, altering microtubule dynamics and changing tubulin subtypes, compositions, and posttranslational modifications (5).

Histone deacetylase 6 (HDAC6) is a special enzyme with a unique structure and various functions. It possesses two deacetylase domains and is involved in various deacetylase-dependent and -independent cytoplasmic cellular processes, such as inflammation, angiogenesis, transcription, protein degradation, cell survival, and cell motility (6-8). HDAC6 targets various substrates, including $\alpha$-tubulin, cortactin, HSP90, Tat, Ku70, $\beta$-catenin, peroxiredoxins, and survivin (8). The overexpression of HDAC6 induces tumorigenesis in mammalian cells by hypoacetylating $\alpha$-tubulin and promoting the formation of immune synapses, cell migration, and chemotaxis. As a result, extensive studies have been conducted to develop HDAC6-selective inhibitors to target tumorigenesis and metastasis. One of the HDAC6-specific inhibitors, ACY-1215 (ricolinostat), is under a phase1b clinical trial for relapsed or refractory multiple myeloma (9). Moreover, HDAC6 inhibition through ACY-1215 was proven to be effective in various cancers, including inflammatory and metastatic breast cancers, melanoma, glioblastoma, prostate cancer, colorectal cancer, multiple myeloma, and lymphoma (9-16). ACY-241 or citarinostat, like ACY-1215, inhibits HDAC6 with 13 to 18-fold reduced potency against HDAC1, 2, and 3 isozymes (17). However, as a second generation inhibitor, ACY-241 carries improved solubility compared to its structurally related inhibitor ACY-1215 and is orally available. Currently, it is under investigation in a clinical trial (NCT02886006) for multiple myeloma. Apart from ACY-1215 and ACY-241, A452 is a potent hydroxamic acid based HDAC6-selective small-molecule inhibitor under preclinical studies $(18,19)$. A452 carries a $\gamma$-lactam core that selectively inhibits HDAC6 catalytic activity and successfully suppresses colorectal cancer cell growth. However, further research is required to evaluate its efficacy and mechanisms in other cancers, such as in epithelial ovarian carcinomas (EOC).

Approximately $85-90 \%$ of malignant ovarian cancer subtypes are EOCs. They are classified histologically into four types: high- and low-grade serous carcinomas account for $75 \%$ of EOCs, whereas clear cell carcinomas, mucinous carcinomas, endometrioid carcinomas comprise 9,3 and $8 \%$ of EOCs, respectively. EOCs are known to carry mutually exclusive ARIDIA and TP53 mutations (20). ARIDIA, a component of the SWI/SNF complex, is mutated in more than $50 \%$ of ovarian clear cell carcinomas and $30 \%$ of ovarian endometrioid carcinomas. These ARIDIA-mutated ovarian cancers fail to inhibit HDAC6 activity, thus destabilizing p53 by removing its lysine 120 acetylation (K120Ac) (21). This increased HDAC6 activity promotes tumor growth. Therefore, using a selective HDAC6 inhibitor, such as ACY-1215, in ARID1A-inactivated ovarian cancer cells induces apoptosis by modulating p53 stability.

In this study, we conducted further experiments using two different HDAC6-selective inhibitors, ACY-241 and A452, to confirm the anticancer effect of HDAC6 inhibition on ARID1A-null ovarian cancer TOV-21G cells with a wild type TP53. Furthermore, as both HDAC6 and PTX alter cellular $\alpha$-tubulin dynamics, the combination treatment of PTX and HDAC6 inhibitors synergistically enhances apoptosis in TOV-21G cancer cells. This may be a novel therapeutic strategy in overcoming the limitations of PTX.

\section{Materials and methods}

Ovarian cancer cell lines and culture. The human ovarian clear cell carcinoma cell, TOV-21G, was purchased from American Type Culture Collection (ATCC). It was cultured in Dulbecco's modified Eagle's medium (Sigma-Aldrich; Merck KGaA) containing 10\% fetal bovine serum (HyClone; GE Healthcare), $100 \mathrm{U} / \mathrm{ml}$ penicillin and $100 \mu \mathrm{g} / \mathrm{ml}$ streptomycin (Gibco; Thermo Fisher Scientific, Inc.) in a $5 \% \mathrm{CO}_{2}$ and $37^{\circ} \mathrm{C}$ incubator.

Cell growth and viability assay. As described elsewhere (14), to measure cell growth and viability, the cells were seeded in triplicates at a density of $3 \times 10^{3}$ cells in $130 \mu \mathrm{l}$ of medium in 96 -well plates. Following $24 \mathrm{~h}$ of incubation, drugs were added to each well, then incubated for 24,48 and $72 \mathrm{~h}$ each. Following the drug treatment, $13 \mu \mathrm{l}$ of CCK-8 reagent (WST-8; CCK-8 kit, CK04; Dojindo Molecular Technologies, Inc.) was added into each well, and the plate was incubated at $37^{\circ} \mathrm{C}$ for $3 \mathrm{~h}$. Then, absorbance was read at $450 \mathrm{~nm}$ using microplate reader (Tecan Group, Ltd.). The results are presented as percentages relative to the control wells. All half-maximal growth inhibition concentration $\left(\mathrm{GI}_{50}\right)$ and half-maximal inhibitory concentration $\left(\mathrm{IC}_{50}\right)$ values were calculated using GraphPad Prism ver. 7.0 software.

Colony formation assay. To examine colony forming ability, the cells were seeded at 6 -well plates at a density of $1 \times 10^{3}$ cells in $2 \mathrm{ml}$ of medium. Following $24 \mathrm{~h}$ of incubation, drugs were treated in each wells, and the plate was incubated at $37^{\circ} \mathrm{C}$ for 14 days. The medium with drugs were renewed every 3 to 4 days. The cells were stained with $0.7 \mathrm{ml} 0.05 \%$ crystal violet solution (Sigma-Aldrich; Merck KGaA) for $30 \mathrm{~min}$ and the colonies were counted using open-source software OpenCFU (22). The experiment was carried out in triplicates. One-way ANOVA with Bonferroni's post-hoc test was carried out using GraphPad Prism 7.0 software (GraphPad Software).

Drug combination analysis. To determine drug combination effects, constant ratio of PTX to ACY-241 and PTX to A452 were used. Cells were seeded in triplicates in 96-well plates at the density of $3 \times 10^{3}$ cells. The plate was incubated overnight before adding drugs of diluted concentrations prior to use. The cells were incubated with the drugs for $72 \mathrm{~h}$ at $37^{\circ} \mathrm{C}$. A CCK-8 assay was performed to determine cell viability. Drug synergy was determined according to the combination index (CI) method described by Chou (23); $\mathrm{CI}<1$ implies synergism, $\mathrm{CI}=1$ implies additivity, CI $>1$ implies antagonism. CI values were calculated using CalcuSyn software version 2.11 (Biosoft). Two-way ANOVA with Bonferroni's post-hoc test for both combination was conducted using GraphPad Prism 7.0 software (GraphPad Software).

Wound healing assay. To determine wound closure capability, the cells were seeded in 6 -well plates at density of $1 \times 10^{6}$ cells in triplicates. The cells were incubated in DMEM culture medium containing $10 \%$ fetal bovine serum for $24 \mathrm{~h}$. After 
24-h incubation at $37^{\circ} \mathrm{C}$, the wound was scratched using 1,000 pipet tip. Then, the cells were washed to remove cell debris and were treated with drugs for $24 \mathrm{~h}$. The cells were removed from the incubator and were washed with $1 \mathrm{ml}$ of medium and photographed. The wound was measured using ImageJ software. One-way ANOVA with Bonferroni's post-hoc test was carried out using GraphPad Prism 7.0 software (GraphPad Software).

Western blot analysis. TOV-21G cells were seeded in 6-well plates at density of $5 \times 10^{5}$ cells/well and incubated for overnight in $37^{\circ} \mathrm{C}$ before drug treatment. After drug treatment, cells were incubated for $24 \mathrm{~h}$. Cells were lysed and extracted with $100 \mu \mathrm{l}$ NP-40 lysis buffer [0.5\% NP-40, 50 mM Tris-HCl (pH 7.4), $120 \mathrm{mM} \mathrm{NaCl}, 25 \mathrm{mM} \mathrm{NaF}, 25 \mathrm{mM}$ glycerol phosphate, $1 \mathrm{mM}$ EDTA, $5 \mathrm{mM}$ EGTA, $1 \mathrm{mM}$ PMSF and $1 \mathrm{mM}$ bezamidine] and sonicated twice for $3 \mathrm{sec}$ at $20 \%$ amplitude. Bradford protein assay was conducted to measure protein concentrations. Cell lysates containing $60-80 \mu \mathrm{g}$ proteins were loaded onto $7.5-12 \%$ SDS-PAGE. Then, proteins were transferred to nitrocellulose membrane at $100 \mathrm{~V}$ for $100 \mathrm{~min}$. Membranes were blocked with $5 \%$ skim milk at room temperature and were incubated for overnight with primary antibodies at $4^{\circ} \mathrm{C}$. Primary antibodies were diluted using following concentrations: $\alpha$-tubulin (1:1,000), acetyl- $\alpha$-tubulin (1:2000), GAPDH (1:10,000), PARP (1:1,000), Pro-caspase-3 (1:1,000), cleaved caspase-3 (1:500), Pro-caspase-8 (1:500), Pro-caspase-9 (1:500), Bak (1:1,000), Bax (1:500), Bcl-xL (1:1,000), Bcl-2 (1:500), p53 (1:1,000), p53K120Ac (1:500), p53K381Ac $(1: 1,000)$, and p-p53ser15 (1:500). Then membranes were washed with $0.1 \%$ Tween-20/ PBS and incubated with anti-rabbit/mouse secondary antibody coupled to HRP at room temperature for $3 \mathrm{~h}$. Protein bands were detected with ECL western blotting solution (Thermo Scientific Pierce).

Apoptosis assay. Apoptosis assay was performed with Annexin V/propidium iodide (PI) double staining. Initially, cells were seeded in $100 \Phi$ plates at densities of $1.2 \times 10^{6}-1.5 \times 10^{6}$ cells. Cells were incubated for overnight in $37^{\circ} \mathrm{C}$ and treated with drugs accordingly. Drug treated cells were incubated for $48 \mathrm{~h}$, washed with ice-cold PBS and detached with trypsin-EDTA. Cells were collected after centrifugation at 1,000 $\mathrm{rpm}$ for $3 \mathrm{~min}$, then resuspended with 400-700 $\mu \mathrm{l}$ binding buffer. Cells were stained with $5 \mu \mathrm{l} \mathrm{PI}$ and $0.7 \mu \mathrm{l}$ Annexin V-fluorescein isothiocyanate (FITC) in dark for $15 \mathrm{~min}$. The cells were diluted with $400 \mu \mathrm{l}$ of $1 \mathrm{X}$ binding buffer (Annexin V-FITC Apoptosis Detection Kit; BD556547; BD Pharmingen). Finally, the stained cells were analyzed using a flow cytometer and BD FACSDiva software version 7 (both from BD Biosciences). One-way ANOVA with Bonferroni's post-hoc test was carried out using GraphPad Prism 7.0 software (GraphPad Software).

Statistical analysis. All data in this research are presented as means \pm standard deviation of or more than three independent experiments. One-way or two-way ANOVA followed by a Bonferroni's multiple comparison post-hoc analysis was conducted using GraphPad Prism software 7.0 (GraphPad Software). P-value $<0.05$ was considered statistically significant for all data presented.
Table I. Drug inhibition concentrations of TOV-21G cells treated with PTX and histone deacetylase 6-selective inhibitors.

\begin{tabular}{lccc}
\hline & \multicolumn{3}{c}{ Drug $(48 \mathrm{~h})$} \\
\cline { 2 - 4 } Variable & PTX,nM & ACY-241, $\mu \mathrm{M}$ & A452, $\mu \mathrm{M}$ \\
\hline $\mathrm{GI}_{50}$ & 28.74 & 8.89 & 0.69 \\
$\mathrm{IC}_{50}$ & 33.61 & 9.13 & 0.80 \\
\hline
\end{tabular}

Cell growth and cell viability curves were calculated using the curves generated in Fig. 1. Data are presented as the means of three independent experiments. $\mathrm{GI}_{50}$, drug concentration that inhibited $50 \%$ of cell growth; $\mathrm{IC}_{50}$, drug concentration that inhibited $50 \%$ of cell viability; PTX, paclitaxel.

\section{Results}

PTX and the two HDAC inhibitors, ACY-241 and A452, reduce cell growth and cell viability. PTX is a taxane-based chemotherapeutic drug known to inhibit the cell growth of various tumors (3). Similarly, ACY-241 and A452, both HDAC6-selective inhibitors, are known to suppress cell growth and decrease cell viability in solid tumors (17), multiple myeloma $(24,25)$ and colorectal cancer $(18,19)$. To examine the anticancer effects of PTX and the two HDAC6 inhibitors in ovarian cancer cells, we measured the cell growth and viability of TOV-21G cells treated with PTX, ACY-241 and A452. PTX effectively reduced the cell growth and viability of TOV-21G in both a time- and dose- dependent manner (Fig. $1 \mathrm{~A}$ and $\mathrm{B}$ ). When the $\mathrm{GI}_{50}$ and $\mathrm{IC}_{50}$ were calculated, PTX inhibited cell growth at $28.74 \mathrm{nM}$ and reduced cell viability at $33.61 \mathrm{nM}$ (Table I). On the other hand, compared to PTX, the HDAC6 inhibitor ACY-241 inhibited cell viability and growth at higher concentrations with a $\mathrm{GI}_{50}$ value of $8.89 \mu \mathrm{M}$ and an $\mathrm{IC}_{50}$ of $9.13 \mu \mathrm{M}$ (Fig. 1C and D). Compared to ACY-241, A452 had 12 times lower $\mathrm{GI}_{50}$ and 11 times lower $\mathrm{IC}_{50}$ in TOV-21G (Fig. 1E and F). The $\mathrm{GI}_{50}$ value of $\mathrm{A} 452$ was $0.69 \mu \mathrm{M} \mathrm{IC}_{50}$ value of $\mathrm{A} 452$ was $0.8 \mu \mathrm{M}$. PTX was the most effective drug among the three drugs, and A452 was more effective in reducing cell viability than ACY-241.

Combination treatment of PTX and HDAC6-selective inhibitors synergistically decrease cell viability and inhibit cell growth in ovarian cancer cells. Next, we employed the combination therapy of PTX and ACY-241 or PTX and A452 on TOV-21G and analyzed its effects on cell viability and cell growth using Cell Counting Kit (CCK)-8 assays. Synergy was evaluated by calculating CI values based on the Chou and Talalay method (23). The combination treatment of PTX and ACY-241 at a dose ratio of 1:1,000 resulted in CI values $<1$, with PTX and ACY-241 concentrations of 3, 4, 5 and $6 \mathrm{nM}$ and 3, 4, 5 and $6 \mu \mathrm{M}$, respectively (Fig. 2A). On the other hand, the combination treatment of PTX and A452 at a dose ratio of 1:400 resulted in CI values less than 1 from concentrations of $0.25 \mathrm{nM}$ PTX and $0.1 \mu \mathrm{M}$ A452 up to $8 \mathrm{nM}$ PTX and $3.2 \mu \mathrm{M}$ A452 (Fig. 2B). As single treatment of A452 is more effective than ACY-241 in inhibiting cell growth and reducing cell viability in TOV-21G, PTX synergized with A452 at 

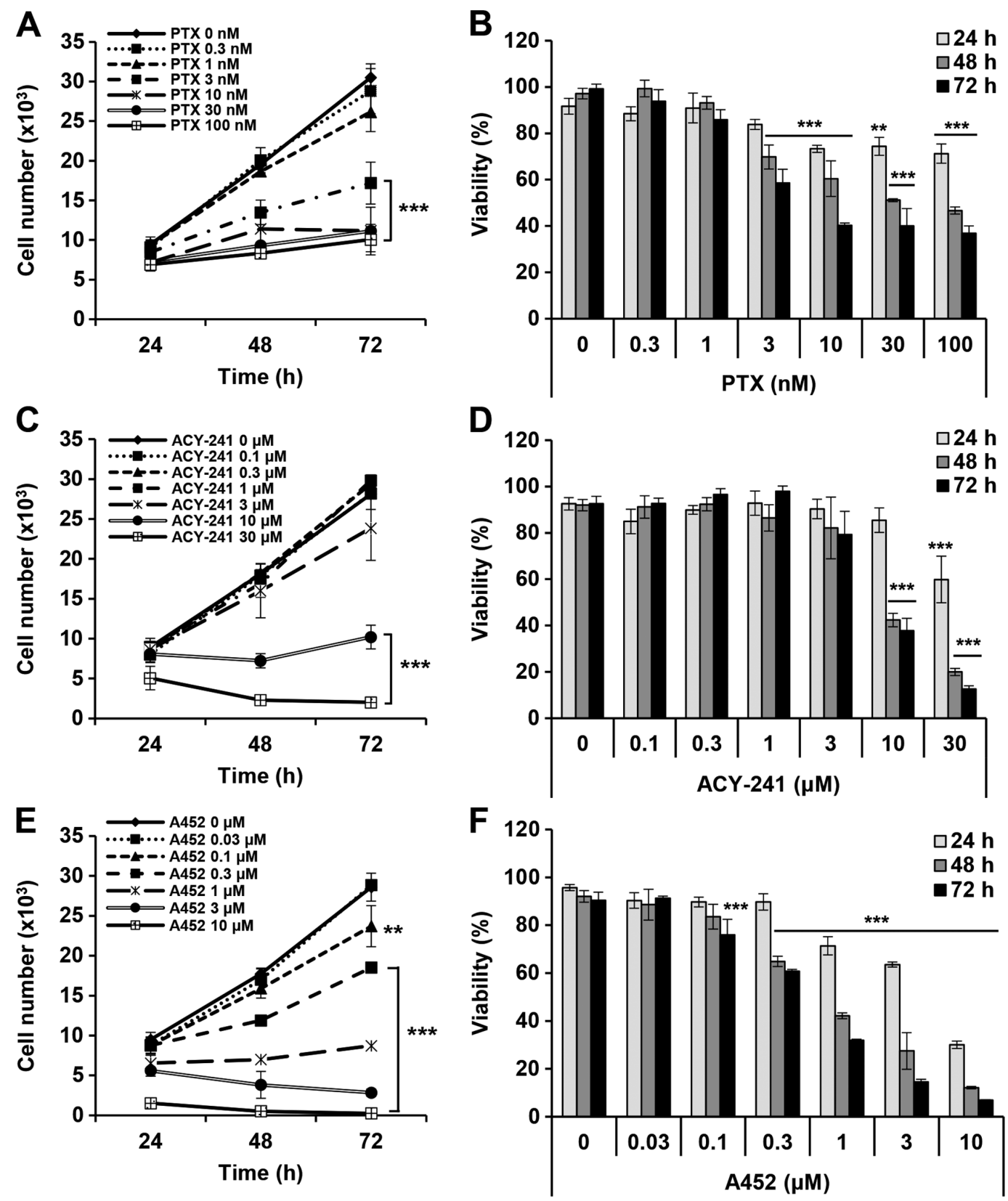

Figure 1. PTX, ACY-241 and A452 individually suppress the growth and viability of the AT-rich interaction domain 1A-null, p53-wild-type TOV-21G ovarian cancer cell line. (A and B) PTX $(0,0.1,1,3,10,30$ and $100 \mathrm{nM})$, (C and D) ACY-241 $(0,0.1,0.3,1,3,10$ and $30 \mu \mathrm{M})$ and $(\mathrm{E}$ and F) A452 (0, $0.1,0.3,1,3,10$ and $30 \mu \mathrm{M}$ ) were used for treatment of TOV-21G cells for 24,48 and $72 \mathrm{~h}$ at the respective concentrations. (A, C and E) Cell growth and (B, D and F) cell viability were measured using Cell Counting Kit-8 assays in 96-well plates. All three drugs inhibited the growth and decreased the viability of TOV-21G cells. Cell counts were estimated indirectly from a standard curve derived from absorbance of known cell numbers. The absorbance at each concentration was normalized to that of the negative control within the corresponding time interval. Data are presented as the mean $\pm \mathrm{SD}(\mathrm{n}=3){ }^{* * *} \mathrm{P}<0.01$ and ${ }^{* * * *} \mathrm{P}<0.001$ vs. the DMSO control (one-way ANOVA with Bonferroni's post hoc test; the significance levels apply to all samples under a line). PTX, paclitaxel.

substantially lower doses than it did with ACY-241. The long-term combination treatment of PTX and both HDAC6 inhibitors also significantly inhibited the colony-forming abilities of TOV-21G (Fig. 2C and D). Moreover, as HDAC6 inhibition is known to suppress migration and metastasis in various cancers $(26,27)$, wound healing assays were conducted to examine the effects of PTX and the two HDAC6-selective inhibitors (Fig. 3A and B). Both drug combinations synergistically suppressed TOV-21G migration and proliferation.

PTX and HDAC6-selective inhibitors synergistically result in apoptosis in ovarian cancer cells. To further confirm the synergy of PTX and HDAC6-selective inhibitors, we performed a western blot analysis to assess drug synergy at the protein level. Several apoptotic proteins were activated on the combination treatment of PTX with ACY-241 (Fig. 4A). Poly(ADP-ribose) polymerase (PARP), which is involved in DNA repair, genomic stability, and programmed cell death, was synergistically cleaved on combination treatment, resulting in apoptosis. As a PARP regulating protein, cleaved caspase-3 was also significantly increased upon combination treatment. On the other hand, Bcl-2 family related pro-apoptotic proteins such as Bak and Bax did not show significant changes in expression levels. However, Bcl-2 family-related anti-apoptotic 
A

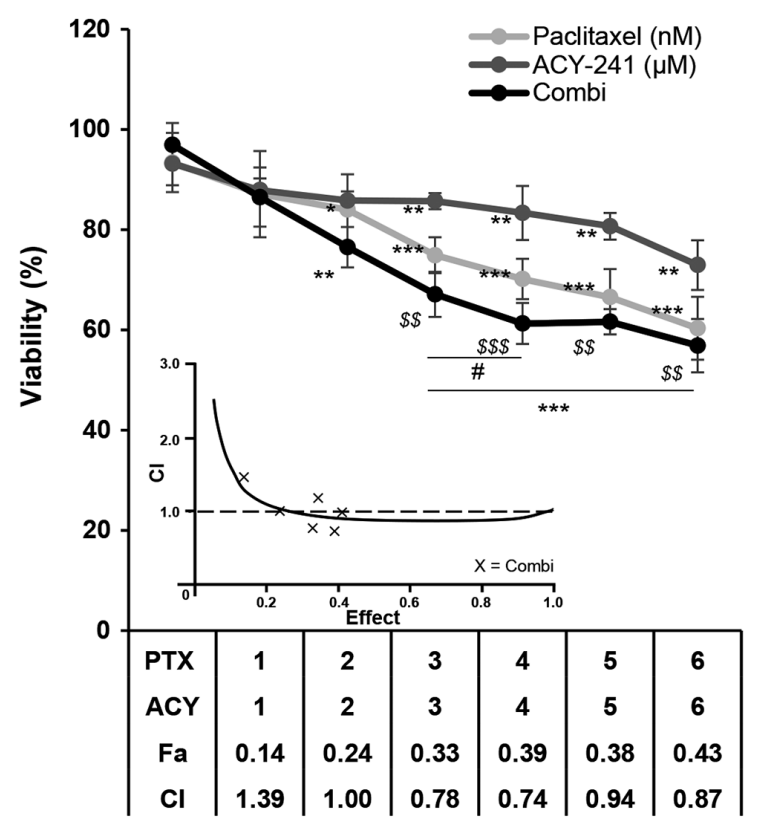

B

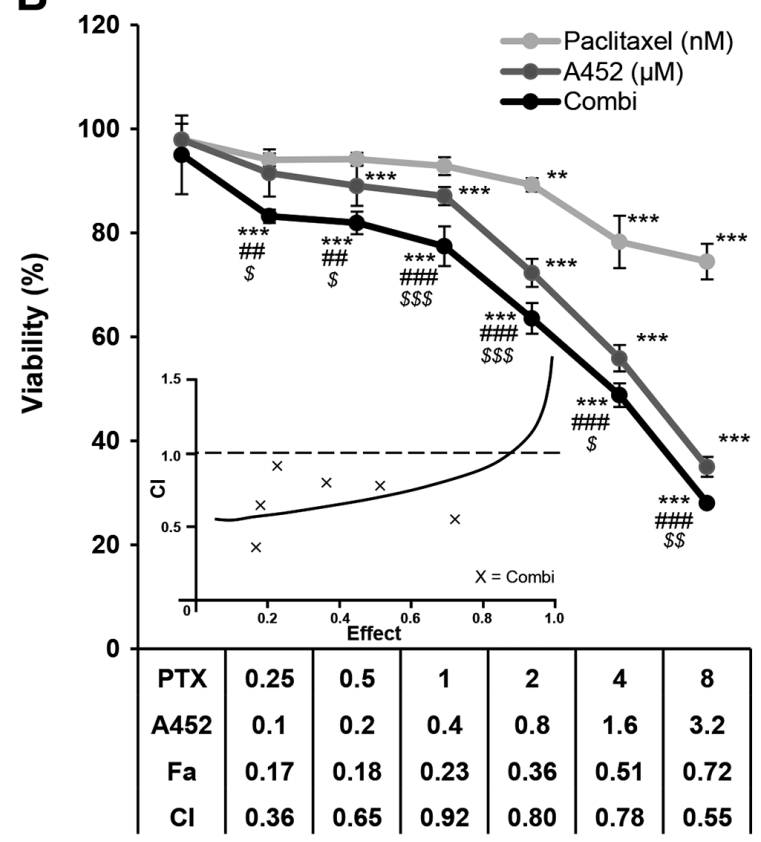

C
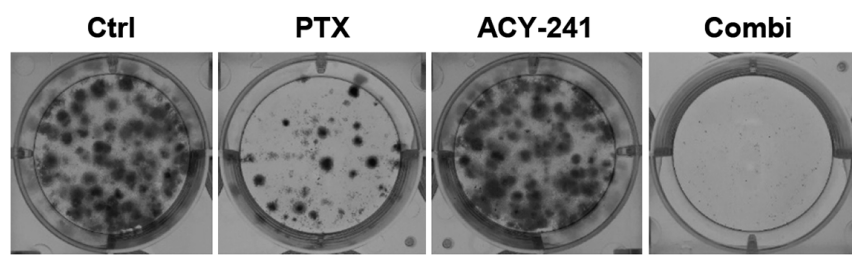

D
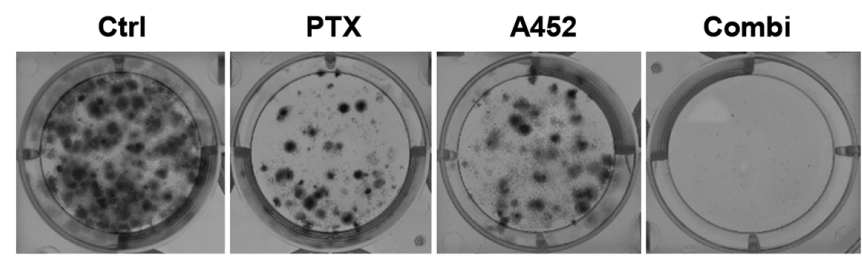
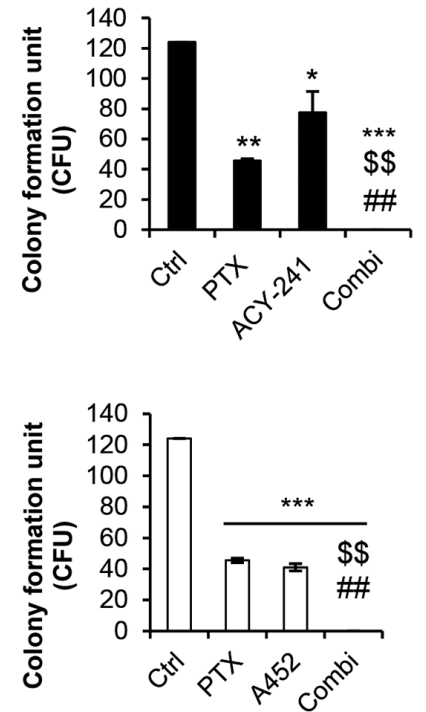

Figure 2. PTX and HDAC6-selective inhibitors synergistically decrease cell viability and proliferation in the short and long term. (A) TOV-21G cells were treated with $0.1 \%$ DMSO control, PTX, ACY-241 or a combination of these agents for $72 \mathrm{~h}$. (B) TOV-21G cells were treated with $0.1 \%$ DMSO control, PTX, A452 or a combination of these agents for $72 \mathrm{~h}$. PTX and both HDAC6-selective inhibitors synergistically reduced cell viability. (C) Combination treatment of PTX and ACY-241 synergistically inhibited the colony formation of TOV-21G cells. (D) Combination treatment of PTX and A452 synergistically inhibited the colony formation of TOV-21G cells. The drug concentrations used for colony formation assays were as follows: $0.1 \%$ DMSO, $4 \mathrm{nM} \mathrm{PTX,} 0.2 \mu \mathrm{M}$ ACY-241 and $0.1 \mu \mathrm{M}$ A452. Data are presented as the mean $\pm \mathrm{SD}(\mathrm{n}=3)$. ${ }^{*} \mathrm{P}<0.05,{ }^{* *} \mathrm{P}<0.01$ and ${ }^{* * * *} \mathrm{P}<0.001$ vs. DMSO control; ${ }^{\$} \mathrm{P}<0.05,{ }^{\$ \$} \mathrm{P}<0.01$ and ${ }^{\$ \$ \$} \mathrm{P}<0.001 \mathrm{vs}$. $\mathrm{P} T \mathrm{XX}$ ${ }^{\#} \mathrm{P}<0.05,{ }^{\# \#} \mathrm{P}<0.01$ and ${ }^{\# \# /} \mathrm{P}<0.001$ vs. ACY-241 or A452, using a two-way ANOVA test for (A) and (B), and one-way ANOVA tests were used for (C) and (D). ACY, ACY-241; Combi, combination treatment; Fa, fraction affected; CI, combination index; PTX, paclitaxel; CFU, colony-forming unit; Ctrl, control; HDAC6, histone deacetylase 6.

proteins such as Bcl-xL and Bcl-2, decreased synergistically after the combination treatment of PTX and ACY-241, thus supporting the synergism between the two drugs. Similarly, when PTX and A452 were treated together, both cleaved PARP and cleaved caspase-3 increased synergistically (Fig. 4B). However, in this combination, pro-apoptotic proteins Bak and Bax synergistically increased, and anti-apoptotic proteins Bcl-xL and Bcl-2 synergistically decreased. These results further suggest that the combination of PTX and A452 has greater synergy than that of PTX and ACY-241.
To further examine the apoptotic effect of the HDAC6 inhibitors and PTX, we performed an apoptosis assay using a flow cytometry analysis system (Fig. 5A and B). The percentage of apoptotic cells increased synergistically in both drug combinations, as seen in the bar graph. The combination treatment of PTX and ACY-241 increased the percentage of apoptotic cells to $30 \%$, whereas the control (dimethyl sulfoxide [DMSO] 0.1\%), PTX, and ACY-241 remained at 4, 11 and 7\%, respectively (Fig. 5A). Furthermore, the combination treatment of PTX and A452 increased the percentage of 

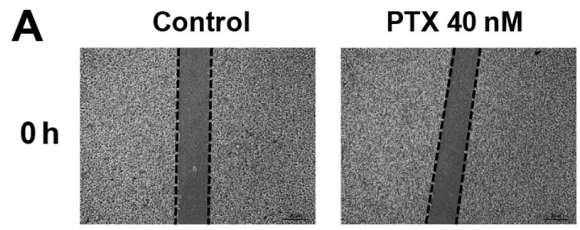

$24 \mathrm{~h}$

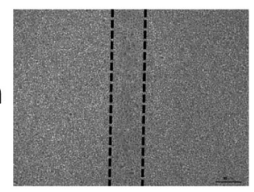

B
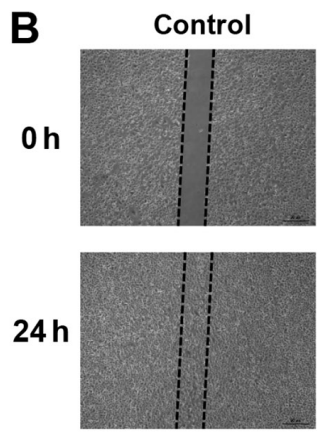
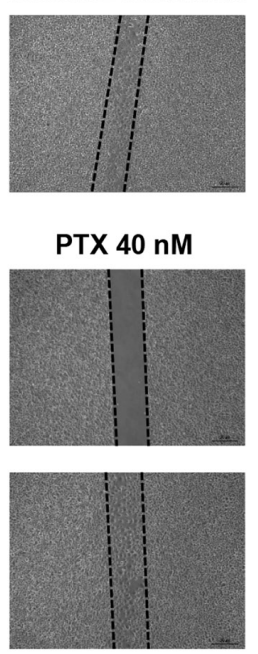
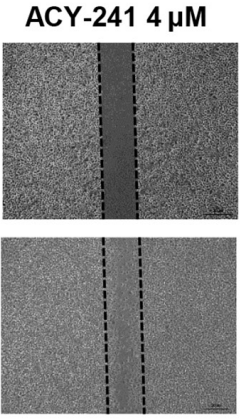

A452 $2 \mu \mathrm{M}$
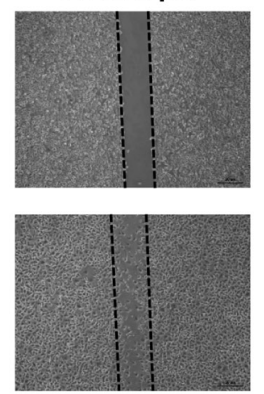
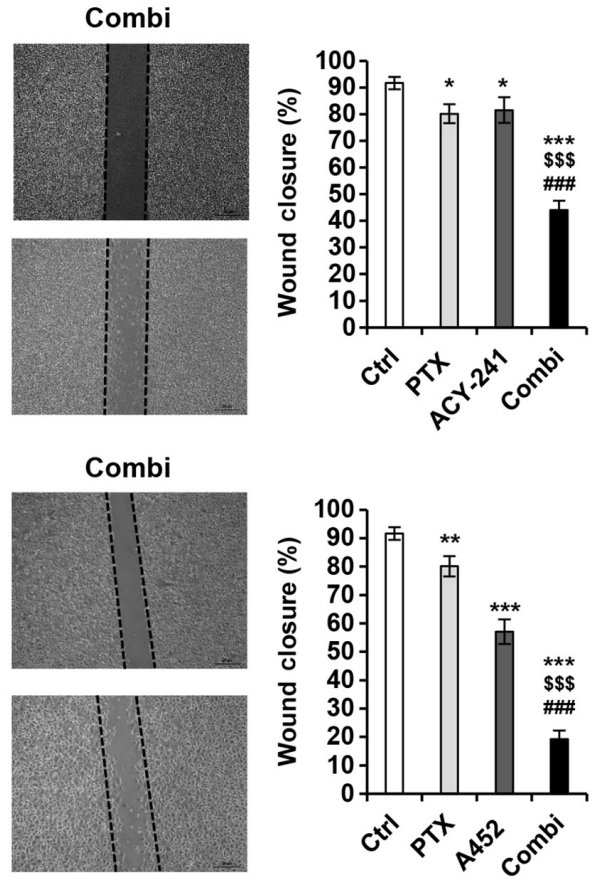

Figure 3. Combination treatments of PTX and histone deacetylase 6-selective inhibitors synergistically suppress the proliferation and migration of TOV-21G cells. (A) PTX (40 nM) and ACY-241 (4 $\mu \mathrm{M})$ significantly inhibited the wound closure of TOV-21G cells compared with either drug alone. (B) PTX (40 nM) and A452 $(2 \mu \mathrm{M})$ significantly inhibited the wound closure of TOV-21G cells compared with either drug treatment as monotherapy. Magnification, $\mathrm{x} 50$. Data are presented as the mean $\pm \mathrm{SD}(\mathrm{n}=3)$. ${ }^{*} \mathrm{P}<0.05,{ }^{* *} \mathrm{P}<0.01$ and ${ }^{* * * *} \mathrm{P}<0.001$ vs. DMSO control; ${ }^{\$ \$} \mathrm{P}<0.001$ vs. PTX; ${ }^{\# \#} \mathrm{P}<0.001$ vs. ACY -241 or A452 (one-way ANOVA). Ctrl, control; Combi, combination treatment; PTX, paclitaxel.

cell apoptosis to $61 \%$. The percentages of cell apoptosis in the control group (DMSO 0.1\%) and in monotherapies of PTX and A452 remained at 6, 19 and 36\%, respectively (Fig. 5B). Corresponding to the apoptotic protein levels, A452 and PTX resulted in greater apoptosis than ACY-241 and PTX. These findings indicate that the combination treatment of PTX and HDAC6-selective inhibitors synergistically induces apoptosis in ovarian cancer cells.

Combination treatment of PTX and HDAC6-selective inhibitors increases p53 stability synergistically. As HDAC6 inhibition is known to increase the lysine acetylation of p53 $(21,28)$, we performed a western blot analysis to determine whether co-treatment of PTX and HDAC6 inhibitors would affect p53 modification levels. Acetylated- $\alpha$-tubulin was used as a positive control to confirm the presence of PTX and HDAC6 inhibitors (Fig. 6A and B). The single treatment of PTX did not increase p53 lysine (K120, K381) acetylation levels in both combinations (Fig. 6A and B). However, co-treatment of PTX and both HDAC6 inhibitors significantly increased acetylation, compared to the individual monotherapy of the three drugs. Thus, it shows that the combination treatment of PTX and ACY-241 or PTX and A452 increases p53 stability synergistically, inducing the apoptosis of ovarian cancer cells. Despite the increase in p53 stability, however, p53 levels did not correlate with the increase in protein stability levels. Such trend can be explained by the phosphorylation of p53 at serine 15 , which seems to play a greater role in determining protein levels of p53 directly. In fact, the phosphorylation levels of p53 correlate positively with p53 protein levels, as PTX monotherapy increases phosphorylated p53 levels to a greater degree than the HDAC6 inhibitors either in combination or as monotherapy. PTX treatment and HDAC6 inhibition may alter p53 levels post-transcriptionally and induce apoptosis in TOV-21G.

\section{Discussion}

Since its discovery, PTX has been used widely to treat various types of solid tumors (3), including ovarian cancer (2). Monotherapies and combination therapies using PTX in ovarian cancer are being used to treat EOCs and are under various clinical studies. For example, PTX was added to cisplatin in the first-line treatment of EOCs, and PTX combined with carboplatin has been the standard treatment for more than 20 years $(2,29)$. In addition to the trials in combination with platinum-based drugs, PTX with bevacizumab in recurrent and platinum-sensitive ovarian cancer is under a phase III clinical trial (30). However, incomplete clinical responses exist, and advanced ovarian cancer remains incurable. Thus, there is a need to develop improved, tolerable chemotherapeutic methods using novel drugs to treat EOCs.

HDACs are essential epigenetic enzymes that regulate various cellular pathways. HDAC inhibitors have been used in multiple studies as alternatives to treat cancer; these induce cell death, cell cycle arrest, and senescence in tumor cells $(31,32)$. Pan-HDAC inhibiting agents, such as butyrate, romidepsin, and vorinostat are under various stages of clinical investigation to treat various cancers. To date, vorinostat and romidepsin have been approved by the FDA to treat cutaneous T-cell lymphoma and peripheral T-cell lymphoma (32). Despite the various ongoing studies, however, pan-HDAC inhibitors elicit side effects because they lack the specificity for individual HDAC isoforms. Therefore, more tolerable 

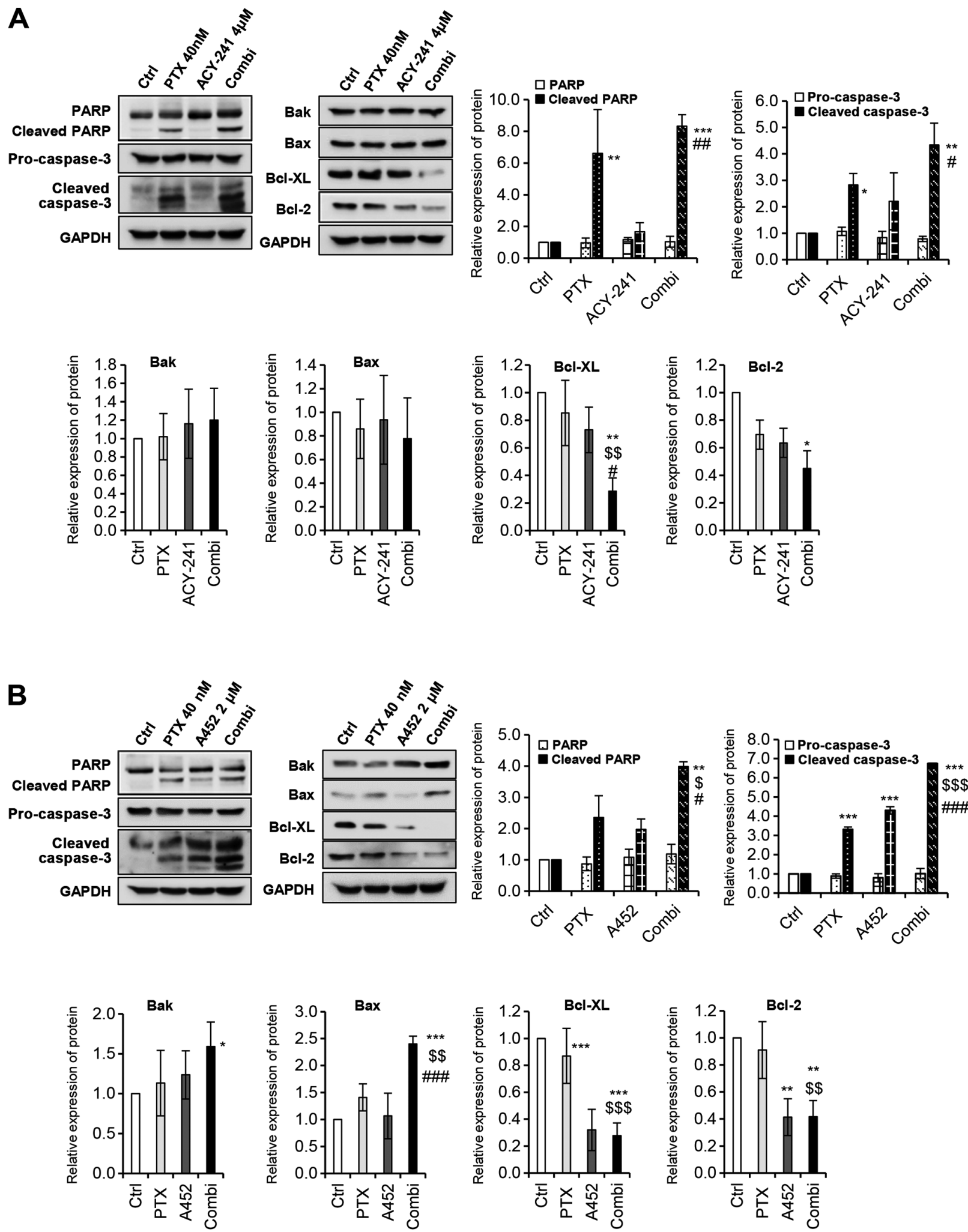

Figure 4. PTX and histone deacetylase 6-selective inhibitors synergistically induce and reduce pro-/anti-apoptotic markers. (A) Combination treatment of PTX $(40 \mathrm{nM})$ and ACY-241 $(4 \mu \mathrm{M})$ significantly increased pro-apoptotic markers while decreasing anti-apoptotic markers. (B) Combination treatment of PTX $(40 \mathrm{nM})$ and A452 $(2 \mu \mathrm{M})$ significantly induced pro-apoptotic markers while reducing anti-apoptotic markers. Western blot analysis was performed using the indicated antibodies. The protein expression levels were semi-quantified relative to $\alpha$-tub or GAPDH. The levels in the $0.1 \%$ DMSO group were set as 1 . GAPDH was used as the loading control. Data are presented as the mean $\pm \mathrm{SD}(\mathrm{n}=3)$. ${ }^{*} \mathrm{P}<0.05,{ }^{* * *} \mathrm{P}<0.01$ and ${ }^{* * * *} \mathrm{P}<0.001 \mathrm{vs}$. DMSO control; ${ }^{{ }^{*}} \mathrm{P}<0.05,{ }^{\$} \mathrm{P}<0.01$ and ${ }^{\$ \$ \$} \mathrm{P}<0.001$ vs. PTX; ${ }^{\#} \mathrm{P}<0.05,{ }^{\# \#} \mathrm{P}<0.01$ and ${ }^{\# \# \#} \mathrm{P}<0.001$ vs. ACY-241 or A452 (one-way ANOVA). Bak, Bcl-2 homologous antagonist/killer; Bcl, B-cell lymphoma; Bcl-xL, Bcl-extra large protein; Cas-3, Caspase-3; Ctrl, control; Combi, combination treatment; PARP, poly(ADP-ribose) polymerase; PTX, paclitaxel.

and selective HDAC inhibitors are required, among which HDAC6-selective inhibitors stand as potent anticancer agents.
As a first-in-class selective and potent HDAC6 inhibitor, ACY-1215 is currently being tested in various clinical trials and 

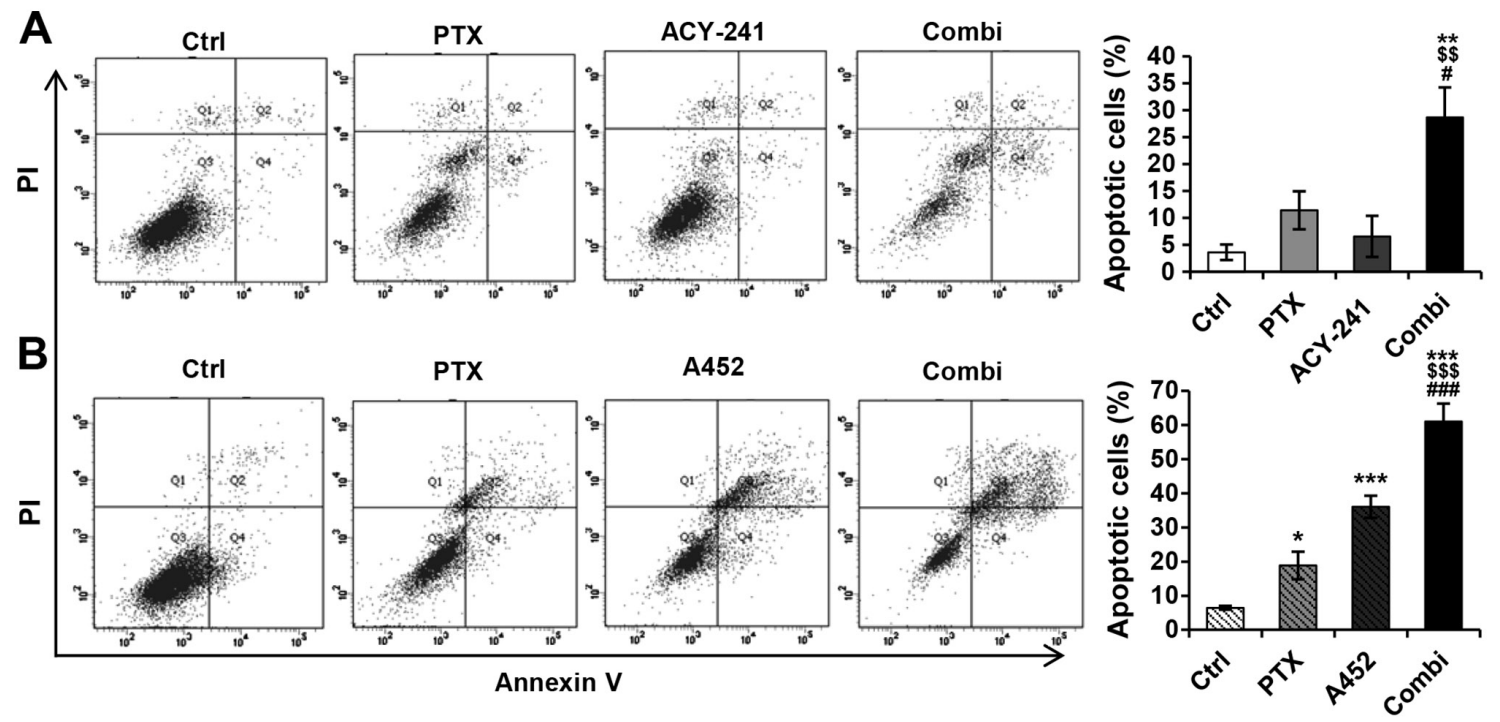

Figure 5. Combination therapy of histone deacetylase 6-selective inhibitors and anticancer agent PTX triggers synergistic apoptosis. (A) TOV-21G cells were treated with $0.1 \%$ DMSO (control), PTX alone, ACY-241 alone and ACY-241 in combination with PTX for $48 \mathrm{~h}$. Whole-cell lysates were prepared and stained with PI and Annexin V. (B) TOV-21G cells were treated with 0.1\% DMSO (control), PTX alone, A452 alone and A452 in combination with PTX for $48 \mathrm{~h}$. Percentages of apoptotic cells were calculated from summation of cells in Q2 and Q4. Data are presented as the mean $\pm \mathrm{SD}(\mathrm{n}=3)$. ${ }^{*} \mathrm{P}<0.05$, ${ }^{* *} \mathrm{P}<0.01$ and ${ }^{* * * *} \mathrm{P}<0.001$ vs. DMSO control; ${ }^{\$ \$} \mathrm{P}<0.01$ and ${ }^{\$ \$ \$} \mathrm{P}<0.001$ vs. PTX; ${ }^{\#} \mathrm{P}<0.05$ and ${ }^{\# \# \#} \mathrm{P}<0.001$ vs. ACY -241 or A452 (one-way ANOVA). Ctrl, control; Combi, combination treatment; PI, propidium iodide; PTX, paclitaxel.

is proven to have antitumor effects in various cancers in monotherapy or in combination with other agents (9-16). Besides ACY-1215, which has been explored widely for its clinical efficacy, other novel HDAC6-selective inhibitors, ACY-241 and A452, need further extensive research. Until now, it is known that ACY-241 (citarinostat) synergizes with pomalidomide in multiple myeloma (24), with PTX in solid tumor models (17), and with anti-PD-L1 antibody in multiple myeloma (25). In addition, previous studies with A452 indicate its potency as an anticancer agent for non-Hodgkin lymphoma (33), multiple myeloma (34), immunomodulatory drugs-resistant multiple myeloma (35), colorectal cancers $(19,28)$, and glioblastoma (36) in vitro.

Previous studies have shown that the combination therapy of HDAC6-selective inhibitor ACY-241 and PTX increased the anticancer drug efficiency of conventionally used PTX in ovarian cancer (17). In this study, we have additionally confirmed the antitumor synergistic effect of PTX and HDAC6 inhibition using both ACY-241 and a novel inhibitor, A452. The co-treatment of PTX and the two HDAC6-selective inhibitors in ARIDIA-null ovarian cancer cells synergistically increased apoptosis (Figs. 4 and 5) and decreased cell growth and viability (Fig. 2). ARIDIA deficient ovarian cancers fail to inhibit HDAC6 activity, which normally deacetylates $\alpha$-tubulin that is crucial for the induction of microtubule disassembly, leading to tumorigenesis. Thus, ARID1A-mutant or -null cells are sensitive to HDAC6 inhibition. Therefore, when PTX, which also inhibits $\alpha$-tubulin polymerization, is taken in combination with HDAC6-selective inhibitors, both $\alpha$-tubulin polymerization and dissociation is inhibited, leading to cell death. The deacetylation of tubulin substrates also regulate microtubule-dependent cell motility, so the inhibition of HDAC6 prevents cell movement and migration (8). Nonetheless, to wholly state the effectiveness of HDAC6-selective inhibitors and PTX in ARID1A-mutant ovarian cancers, further studies using other ovarian cancer cell lines, such as OVISE (clear cell carcinoma, ARID1A mutant, and wild-type TP53), are required. Using both HDAC6 inhibitors and PTX may also impede metastasis in ovarian cancer cells. However, metastasis-related proteins matrix metalloproteinase (MMP)-2 and MMP-9 were not detected (data not shown), suggesting that the two drug combination might prevail in inhibiting proliferation over migration. Although more studies are required to conclusively state the synergistic effect of the two drugs in ovarian cancer metastasis, the inhibition of cell proliferation seems to be evident (Fig. 3). Further studies are required to assess the effects of PTX and HDAC6 inhibition in ovarian cancer cell migration.

Existing studies have demonstrated the synergistic hyperacetylation of $\alpha$-tubulin by PTX and ACY-241 (17). In this study, we demonstrated that A452 also hyperacetylates $\alpha$-tubulin when treated together with PTX (Fig. 6). The research data show that regardless of the type of HDAC6-selective inhibitor used, HDAC6 inhibition and PTX synergistically regulate $\alpha$-tubulin in ARID1A-mutated ovarian cells. In addition to $\alpha$-tubulin regulation, we found that p53 acetylation modifications at lysine 120 and 381 were synergistically increased on combination treatment with PTX using either ACY-241 or A452 (Fig. 6). Although previous studies have shown that PTX and pan-HDAC inhibition using SAHA, ST2785, and ST3595 can also induce the synergistic hyperacetylation of p53 (37), we have shown for the first time that a combination of PTX and HDAC6-selective inhibition increases p53 lysine acetylation. The stabilization of p53 in TOV-21G, which endogenously carries a wild-type p53, resulted in tumor growth suppression. However, extended studies using ARID1A-mutant ovarian cancer cells with wild-type p53 are necessary to strongly confirm that synergistic tumor growth inhibition involves p53. 

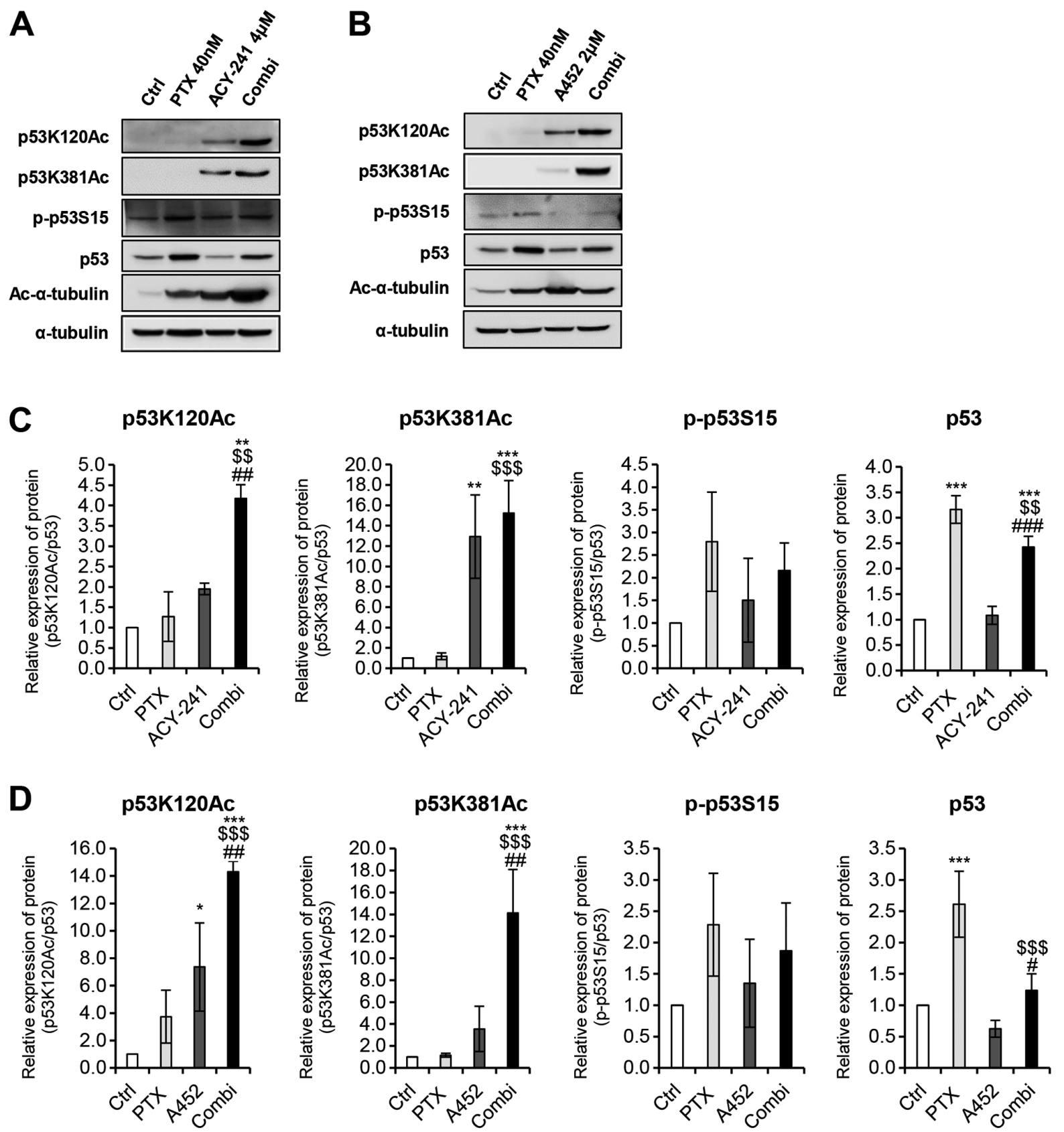

Figure 6. Combined treatment of histone deacetylase 6-selective inhibitors and anticancer agent PTX synergistically alters lysine acetylation modifications of p53. Western blot analysis was performed using the indicated antibodies. (A) Combination treatment of PTX (40 nM) and ACY-241 (4 $\mu$ M) synergistically altered the acetylation modification of p53. (B) Combination treatment of PTX (40 nM) and A452 (2 $\mu \mathrm{M})$ synergistically altered acetylation modification of p53. (C) Relative protein levels of acetylated p53 are shown for combination treatment of PTX (40 nM) and ACY-241 (4 $\mu$ M). (D) Relative protein levels of acetylated p53 are shown for combination treatment of PTX $(40 \mathrm{nM})$ and A452 $(2 \mu \mathrm{M})$. p53 protein expression was semi-quantified relative to $\alpha$-tub; acetylated and phosphorylated $\mathrm{p} 53$ proteins were semi-quantified relative to total $\mathrm{p} 53$ levels. The levels in the $0.1 \%$ DMSO group were set as $1 . \alpha$-tub was used as a loading control. Data are presented as the mean $\pm \mathrm{SD}(\mathrm{n}=3) .{ }^{*} \mathrm{P}<0.05,{ }^{* *} \mathrm{P}<0.01$ and ${ }^{* * *} \mathrm{P}<0.001$ vs. DMSO control; ${ }^{\$ \$} \mathrm{P}<0.01$ and ${ }^{\$ \$ \$} \mathrm{P}<0.001$ vs. $\mathrm{P} T \mathrm{X} ;{ }^{*} \mathrm{P}<0.05$, ${ }^{\# \#} \mathrm{P}<0.01$ and ${ }^{\# \# /} \mathrm{P}<0.001$ vs. ACY-241 or A452, using a one-way ANOVA test. $\alpha$-tub, $\alpha$-tubulin; Ac- $\alpha$-tub, acetylated- $\alpha$-tubulin; Ctrl, control; Combi, combination treatment; PTX, paclitaxel; p53K120Ac, p53 lysine 120 acetylation; p53K381Ac, p53 lysine 381 acetylation; p-p53S15, p53 serine 15 phosphorylation.

Taken together, our data demonstrate the synergistic anticancer activity of PTX and HDAC6-selective inhibitors. As mentioned earlier, HDAC6-selective inhibitors have shown high anticancer potential in different cancers, ranging from blood cancers to solid cancers. In this study, we show that HDAC6-selective inhibition through ACY-241 and A452 is capable of being used in combination with the conventionally used chemotherapeutic drug, PTX, in vitro. Although we did not use clinical samples in our study, our results suggest a proof-of-concept or the preclinical therapeutic possibility of using ACY-241 and A452 with PTX in treating ovarian cancer by activating p53 and inducing apoptosis. Further research is inevitable to elucidate the exact molecular mechanisms behind such synergism in ovarian cancers. Collectively, this study provides beneficial information for clinical trials of combination therapy using HDAC6-selective inhibitors, not only in ovarian cancers but also in other solid tumors. 


\section{Acknowledgements}

Not applicable.

\section{Funding}

The present study was supported by the Basic Science Research Program through the National Research Foundation of Korea funded by the Ministry of Education, Science and Technology (grant nos. 2018R1A6A1A03023718 and 2019R1I1A1A01058601).

\section{Availability of data and materials}

The datasets used and/or analyzed during the current study are available from the corresponding author on reasonable request.

\section{Authors' contributions}

JY and SHK were involved in the general design of the study. JY, DHL and GWK performed the experiments. JY, YHJ, SYK, SWL, JP and SHK analyzed the data. JY, YHJ, DHL, GWK, SYK, SWL, JP and SHK have assessed the authenticity of all raw data. JY wrote the initial draft of the manuscript, JY and SHK extensively edited the manuscript, and SHK supervised the work. All authors read and approved the final manuscript.

\section{Ethics approval and consent to participate}

Not applicable.

\section{Patient consent for publication}

Not applicable.

\section{Competing interests}

The authors declare that they have no competing interests.

\section{References}

1. Dion L, Carton I, Jaillard S, et al: The Landscape and Therapeutic Implications of Molecular Profiles in Epithelial Ovarian Cancer. J Clin Med 9: 2239, 2020.

2. Jayson GC, Kohn EC, Kitchener HC and Ledermann JA: Ovarian cancer. Lancet 384: 1376-1388, 2014.

3. Weaver BA: How Taxol/paclitaxel kills cancer cells. Mol Biol Cell 25: 2677-2681, 2014.

4. Schiff PB, Fant J and Horwitz SB: Promotion of microtubule assembly in vitro by taxol. Nature 277: 665-667, 1979.

5. Orr GA, Verdier-Pinard P, McDaid $\mathrm{H}$ and Horwitz SB: Mechanisms of Taxol resistance related to microtubules. Oncogene 22: 7280-7295, 2003.

6. Boyault C, Sadoul K, Pabion M and Khochbin S: HDAC6, at the crossroads between cytoskeleton and cell signaling by acetylation and ubiquitination. Oncogene 26: 5468-5476, 2007.

7. Cao J, Lv W, Wang L, Xu J, Yuan P, Huang S, He Z and $\mathrm{Hu}$ J: Ricolinostat (ACY-1215) suppresses proliferation and promotes apoptosis in esophageal squamous cell carcinoma via $\mathrm{miR}-30 \mathrm{~d} / \mathrm{PI} 3 \mathrm{~K} / \mathrm{AKT} / \mathrm{mTOR}$ and ERK pathways. Cell Death Dis 9: 817, 2018.

8. Li Y, Shin D and Kwon SH: Histone deacetylase 6 plays a role as a distinct regulator of diverse cellular processes. FEBS J 280: $775-793,2013$
9. Yee AJ, Bensinger WI, Supko JG, Voorhees PM, Berdeja JG, Richardson PG, Libby EN, Wallace EE, Birrer NE, Burke JN, et al: Ricolinostat plus lenalidomide, and dexamethasone in relapsed or refractory multiple myeloma: A multicentre phase $1 \mathrm{~b}$ trial. Lancet Oncol 17: 1569-1578, 2016.

10. Putcha P, Yu J, Rodriguez-Barrueco R, Saucedo-Cuevas L, Villagrasa P, Murga-Penas E, Quayle SN, Yang M, Castro V, Llobet-Navas D, et al: HDAC6 activity is a non-oncogene addiction hub for inflammatory breast cancers. Breast Cancer Res 17: 149, 2015.

11. Peng U, Wang Z, Pei S, Ou Y, Hu P, Liu W and Song J: ACY-1215 accelerates vemurafenib induced cell death of BRAF-mutant melanoma cells via induction of ER stress and inhibition of ERK activation. Oncol Rep 37: 1270-1276, 2017.

12. Li S, Liu X, Chen X, Zhang L and Wang X: Histone deacetylase 6 promotes growth of glioblastoma through inhibition of SMAD2 signaling. Tumour Biol 36: 9661-9665, 2015.

13. Corno C, Arrighetti N, Ciusani E, Corna E, Carenini N, Zaffaroni N, Gatti L and Perego P: Synergistic interaction of histone deacetylase 6- and MEK-inhibitors in castration-resistant prostate cancer cells. Front Cell Dev Biol 8: 610, 2020.

14. Lee DH, Won HR, Ryu HW, Han JM and Kwon SH: The HDAC6 inhibitor ACY 1215 enhances the anticancer activity of oxaliplatin in colorectal cancer cells. Int J Oncol 53: 844-854, 2018.

15. Santo L, Hideshima T, Kung AL, Tseng JC, Tamang D, Yang M, Jarpe M, van Duzer JH, Mazitschek R, Ogier WC, et al: Preclinical activity, pharmacodynamic, and pharmacokinetic properties of a selective HDAC6 inhibitor, ACY-1215, in combination with bortezomib in multiple myeloma. Blood 119: 2579-2589, 2012.

16. Amengual JE, Johannet P, Lombardo M, Zullo K, Hoehn D, Bhagat G, Scotto L, Jirau-Serrano X, Radeski D, Heinen J, et al: Dual targeting of protein degradation pathways with the selective HDAC6 inhibitor ACY-1215 and bortezomib is synergistic in lymphoma. Clin Cancer Res 21: 4663-4675, 2015.

17. Huang P, Almeciga-Pinto I, Jarpe M, van Duzer JH, Mazitschek R, Yang M, Jones SS and Quayle SN: Selective HDAC inhibition by ACY-241 enhances the activity of paclitaxel in solid tumor models. Oncotarget 8: 2694-2707, 2017.

18. Ryu HW, Shin DH, Lee DH, Won HR and Kwon SH: A potent hydroxamic acid-based, small-molecule inhibitor A452 preferentially inhibits HDAC6 activity and induces cytotoxicity toward cancer cells irrespective of p53 status. Carcinogenesis 39: 72-83, 2018.

19. Won HR, Ryu HW, Shin DH, Yeon SK, Lee DH and Kwon SH: A452, an HDAC6-selective inhibitor, synergistically enhances the anticancer activity of chemotherapeutic agents in colorectal cancer cells. Mol Carcinog 57: 1383-1395, 2018.

20. Bitler BG, Wu S, Park PH, Hai Y, Aird KM, Wang Y, Zhai Y, Kossenkov AV, Vara-Ailor A, Rauscher FJ III, et al: ARID1A-mutated ovarian cancers depend on HDAC6 activity. Nat Cell Biol 19: 962-973, 2017.

21. Reed SM and Quelle DE: p53 acetylation: Regulation and consequences. Cancers (Basel) 7: 30-69, 2014.

22. Geissmann Q: OpenCFU, a new free and open-source software to count cell colonies and other circular objects. PLoS One 8: e54072, 2013.

23. Chou TC: Drug combination studies and their synergy quantification using the Chou-Talalay method. Cancer Res 70: 440-446, 2010.

24. North BJ, Almeciga-Pinto I, Tamang D, Yang M, Jones SS and Quayle SN: Enhancement of pomalidomide anti-tumor response with ACY-241, a selective HDAC6 inhibitor. PLoS One 12: e0173507, 2017.

25. Ray A, Das DS, Song Y, Hideshima T, Tai YT, Chauhan D and Anderson KC: Combination of a novel HDAC6 inhibitor ACY-241 and anti-PD-L1 antibody enhances anti-tumor immunity and cytotoxicity in multiple myeloma. Leukemia 32: 843-846, 2018.

26. Kanno K, Kanno S, Nitta H, Uesugi N, Sugai T, Masuda T, Wakabayashi G and Maesawa C: Overexpression of histone deacetylase 6 contributes to accelerated migration and invasion activity of hepatocellular carcinoma cells. Oncol Rep 28: 867-873, 2012.

27. Cho HY, Lee SW, Jeon YH, Lee DH, Kim GW, Yoo J, Kim SY and Kwon SH: Combination of ACY-241 and JQ1 synergistically suppresses metastasis of HNSCC via regulation of MMP-2 and MMP-9. Int J Mol Sci 21: 6873, 2020.

28. Ryu HW, Shin DH, Lee DH, Choi J, Han G, Lee KY and Kwon SH: HDAC6 deacetylates p53 at lysines 381/382 and differentially coordinates p53-induced apoptosis. Cancer Lett 391: 162-171, 2017. 
29. Cong J, Liu R, Hou J, Wang X, Jiang H and Wang J: Therapeutic effect of bevacizumab combined with paclitaxel and carboplatin on recurrent ovarian cancer. J BUON 24: 1003-1008, 2019.

30. Coleman RL, Brady MF, Herzog TJ, Sabbatini P, Armstrong DK, Walker JL, Kim BG, Fujiwara K, Tewari KS, O'Malley DM, et al: Bevacizumab and paclitaxel-carboplatin chemotherapy and secondary cytoreduction in recurrent, platinum-sensitive ovarian cancer (NRG Oncology/Gynecologic Oncology Group study GOG-0213): A multicentre, open-label, randomised, phase 3 trial. Lancet Oncol 18: 779-791, 2017.

31. Falkenberg KJ and Johnstone RW: Histone deacetylases and their inhibitors in cancer, neurological diseases and immune disorders. Nat Rev Drug Discov 13: 673-691, 2014.

32. Mottamal M, Zheng S, Huang TL and Wang G: Histone deacetylase inhibitors in clinical studies as templates for new anticancer agents. Molecules 20: 3898-3941, 2015.

33. Lee DH, Kim GW and Kwon SH: The HDAC6-selective inhibitor is effective against non-Hodgkin lymphoma and synergizes with ibrutinib in follicular lymphoma. Mol Carcinog 58: 944-956, 2019.
34. Won HR, Lee DH, Yeon SK, Ryu HW, Kim GW and Kwon SH HDAC6 selective inhibitor synergistically enhances the anticancer activity of immunomodulatory drugs in multiple myeloma. Int J Oncol 55: 499-512, 2019.

35. Kim GW, Yoo J, Won HR, Yeon SK, Lee SW, Lee DH, Jeon YH and Kwon SH: A452, HDAC6-selective inhibitor synergistically enhances the anticancer activity of immunomodulatory drugs in IMiDs-resistant multiple myeloma. Leuk Res 95: 106398, 2020.

36. Kim GW, Lee DH, Yeon SK, Jeon YH, Yoo J, Lee SW and Kwon SH: Temozolomide-resistant glioblastoma depends on HDAC6 activity through regulation of DNA mismatch repair. Anticancer Res 39: 6731-6741, 2019.

37. Zuco V, De Cesare M, Cincinelli R, Nannei R, Pisano C, Zaffaroni $\mathrm{N}$ and Zunino F: Synergistic antitumor effects of novel HDAC inhibitors and paclitaxel in vitro and in vivo. PLoS One 6: e29085, 2011. 